\title{
Producción masiva forzada de árboles silvestres del desierto de Atacama, Copiapó (Chile)
}

\author{
Mass forced production of wild trees from the Atacama desert, Copiapó (Chile) \\ Bernardo Sepúlveda ${ }^{*}$, Ricardo Santana ${ }^{2}$, Juan Soto V. ${ }^{2}$
}

\begin{abstract}
RESUMEN
En zonas áridas la pérdida de biodiversidad, cobertura y biomasa vegetal son problemas ambientales permanentes. En condiciones naturales áridas, las semillas de especies silvestres suelen germinar irregularmente y en tiempo prolongados, lo que hace difícil usar estas especies en procesos productivos. Se evaluaron condiciones adecuadas para optimizar y masificar la producción desde semillas de especies silvestres arbóreas del valle de Copiapó; usando tratamientos de escariación e inducción hormonal de la germinación con GA3. Se determinó el tratamiento para cada especie y la capacidad de producción efectiva de plántulas. La producción de plantas aumentó $83,8 \%$ sobre la producción normal en vivero antes del proyecto, por incremento de la germinación. Usando el protocolo de producción de diferentes especies se llegó a obtener 25.000 plantas (diferentes especies) semanales, con una proyección anual que permitiría manejar la producción artificial de especies silvestres. La propagación masiva de plantas puede ser una herramienta para conservar poblaciones naturales de especies silvestres y potenciar su uso en procesos productivos.
\end{abstract}

Palabras clave: árboles de zonas áridas, tratamiento pregerminativo, producción inducida.

\begin{abstract}
In arid zones the loss of biodiversity, plant cover and biomass are permanent environmental problems. Under arid natural conditions, seeds of wild species usually germinate in irregular periods and in long time, making it difficult to use these species in productive processes. Suitable conditions for optimizing and producing massively wild trees from seeds of species from the Copiapó Valley were evaluated, using treatments of reaming and hormonal induction of germination with GA3. The induction conditions for each tree species and the effective plant production capacity was determined. Plant production increased $83.8 \%$ on the production of plants in nurseries before the project, by increasing of the germination. Using the production protocol, 25,000 plants from different species by week were produced, with a annual projection which would be possible to control the artificial production of wild plant species. The massive propagation of plants could be a tool to conservation of natural populations of wild species and give potential to use it in production process.
\end{abstract}

Key words: trees from arid zones, pregerminative treatment, massive plant production.

\section{Introducción}

La aridez implica el aumento de temperatura y fuerte caída de precipitación; lo que tiene efecto negativo sobre la biodiversidad (McCarty, 2001), especialmente para las especies endémicas o de ecosistemas frágiles (Milbau et al., 2009), además de afectar a la capacidad de germinación natural de semillas de plantas silvestres. En el Valle de Copiapó ha habido pérdida progresiva de vegetación y aumento de aridez desde antes de 1800 , asociado con el desarrollo industrial; antiguos documentos son de gran valor para comparar las condiciones de la vegetación hasta nuestro tiempo (Phillipi, 1860; Camus, 2004).

La propagación de especies de la flora chilena del norte puede ser una herramienta para la conservación en su territorio original o fuera de poblaciones naturales amenazadas. También puede ser una oportunidad para conservación y difusión de biodiversidad nativa y una posibilidad de uso como cultivos comerciales y para procesos productivos,

1 Universidad de Atacama, Centro Regional de Investigación y Desarrollo Sustentable de Atacama (CRIDESAT), Universidad de Atacama, Copiapó, Región de Atacama, Chile.

2 Corporación Nacional Forestal (CONAF), Copiapó, Región de Atacama, Chile.

* Autor para correspondencia: bernardo.sepulveda@uda.cl.

Fecha de Recepción: 12 Diciembre, 2015.

Fecha de Aceptación: 10 Marzo, 2016. 
sin intervenir las poblaciones naturales. Algunas de las especies en que se ha enfocado el interés son Acacia caven (Mol.) Mol., Aextoxicon punctatum R. et P., Beilschmiedia berteroana (Gay) y B. miersii (Gay), Cordia decandra Hooker et Arnott, Geoffroea decorticans (Gill ex Hook et Arn.) Burkart, entre otras (en http://www.chilebosque.cl/propagación. htlm, diciembre 2014). Actualmente, la Corporación Nacional Forestal (CONAF-Chile) tiene programas específicos para propagar las especies arbóreas locales de Atacama. Contar con metodología para multiplicar especies nativas, independientemente de las condiciones climáticas, permitiría mejorar el servicio ambiental, disminuir el efecto antrópico y la explotación irracional; situación muy delicada y similar a la que sufren las formaciones xerofíticas en Atacama, por lo que también es importante la propagación de especies en peligro (Uribe y Cifuentes, 2004).

La pérdida de biodiversidad quita valor a los recursos fitogenéticos; el procedimiento natural de germinación no compensa el ritmo de uso, manejo o sobreexplotación actual de las especies. El cultivo masivo forzado es una alternativa para producir plantas de calidad y permite que genotipos valiosos puedan ser multiplicados sin costo ambiental para la especie; con producción de plantas en corto tiempo y pudiendo lograrse un reservorio de importancia potencial, ya que la flora local puede tener amplia variedad de usos (Boeri et al., 2000; Marinucci et al., 2004). En Argentina, se ha multiplicado forzadamente Acacia caven, especie con importante tolerancia climática y adaptabilidad ecológica, se la señala como especie colonizadora de lugares degradados (pastoreo, agricultura intensiva, fuego) y, además, tiene una importante variedad de uso en zonas áridas. Las especies de Prosopis en ambiente árido y semiáridos, ofrecen sombra, leña, material de construcción y alimento; por lo que especies sudamericanas son incluidas en programas de reforestación y en sistemas agroforestales silvopastoriles en otros continentes (Vilela \& Ravetta, 2001).

La conservación y uso racional de recursos genéticos vegetales es un importante desafío de carácter global, debido a que el desarrollo implica un adecuado equilibrio entre el uso y la conservación, (Gutiérrez et al., 2003). Lo anterior, centra una problemática en la aplicación de una metodología eficiente que no dañe, sino que promueva las desmedradas formaciones vegetacionales de Atacama. Por ello, el objetivo de este trabajo es evaluar una metodología para producción masiva de especies de plantas nativas y silvestres de la Región de Atacama, para uso tanto natural como comercial.

\section{Materiales y Métodos}

\section{Selección de material vegetal}

En asociación con la Corporación Nacional Forestal (CONAF) se seleccionó plantas arbóreas de Atacama entre Totoral ( $25^{\circ} 53^{\prime} 05^{\prime \prime}$ 'S) y Chañaral (2602 '58"S) y de la línea costera hasta el Tranque Lautaro, 96 $57^{\prime} 49^{\prime \prime}$, incluyendo zonas urbanas y rurales de Copiapó. Se colectó semillas, las muestras se conservaron en sobres de papel y en ambiente fresco a $4{ }^{\circ} \mathrm{C}$, en instalaciones de la CONAF y del CRIDESAT-UDA. El material colectado quedó en custodia de CONAF-Copiapó, como institución a cargo de este tipo de recurso vegetal en Chile.

De las posibilidades regionales, se seleccionó las especies Acacia caven (Molina) Molina, A. capensis Colla, A. saligna (Labill.) Wendl., Prosopis chilensis (Molina) Stuntz, Shinus molle L. y Leucaena leucocefala (Lam.) de Wit.; especies de bajo consumo hídrico y alta rusticidad, características para sobrevivir en zonas áridas, aunque con poblaciones desmedradas, están presentes en la región y son usadas en planes de arborización urbana (Alvarado y Levet, 2014).

\section{Tratamiento pregerminativo}

La escariación libera a las semillas de elementos naturales que le dan resistencia a la entrada de agua. La metodología probada corresponde al ajuste del método conocido que permite hacer a la semilla permeable al agua (escariación) e inducción hormonal de germinación, incluso aplicado mediante intermediación de animales de crianza. Previamente se seleccionó semillas colectadas en terreno, eliminando las demasiado livianas, anembrionadas, perforadas o con algún daño. Para determinar el proceso de escariación, semillas de cada especie se sometieron a varios tiempos de exposición a $\mathrm{HCl}(\mathrm{c})$ (37\%). Luego, se enjuagaron abundantemente con agua corriente para eliminar residuos de ácido. Las semillas escariadas se sometieron a imbibición en solución a diferente concentración de GA3 (ácido giberélico, 200 a 800 ppm). Las semillas se depositaron en papel filtro plegado y, en una cámara húmeda, se observó la 
emergencia de la radícula, lo que se expresó como porcentaje de germinación $(\mathrm{N}=5)$ para cada especie. La inducción se hizo en temporada mayo-junio (invierno) y agosto-septiembre (primavera).

\section{Emergencia inducida de plántulas}

En la imbibición con GA3 de semillas escariadas, las que precipitaron por el peso del agua incorporada se sembraron en almacigueras con capacidad de $13 \times 8$ unidades. Las semillas se sembraron en tres grupos de 38 semillas por especie y por cada tiempo de escariación. Se correlacionó el tiempo de la máxima emergencia de plantas con el tiempo de escariación y tomando en cuenta la estación del año. El grupo control fue grupos de semillas no sometidas ni a escariación ni a inducción hormonal; pero, sometidos a imbibición en agua, paralelamente con los demás tratamientos. Por lo anterior, el tiempo de emergencia de plántulas desde el tratamiento control correspondió al tiempo de emergencia natural de las especies en condiciones de vivero.

Para evaluar los ensayos se registró el tiempo (días) en que la emergencia de plantas viables fue máxima, definida por la aparición completa de los cotiledones. No se registró la germinación en estos ensayos para no interferir la estadística natural de emergencia de plántulas.

\section{Mantención de las plántulas}

Las plántulas se obtuvieron en bandejas de germinación (8x13) en 100\% de turba. Las plantas con desarrollo del primer par de hojas verdaderas fueron transplantadas a bolsas de polietileno negro de $15 \times 25 \mathrm{~cm}(100 \mu)$, con orificios para drenaje. El sustrato estuvo compuesto de arena (1/3) de río, tierra de hojas (1/3) y sustrato limo arcilloso (1/3). Este sustrato se esterilizó por solarización antes de usarse, procedimiento normal en el vivero de CONAF. Las plantas se mantuvieron en sombreadero de malla raschel (80\%), y en condiciones normales climáticas de la región, el régimen de regadío correspondió a la forma usual de la práctica en el vivero.

\section{Ensayo de producción masiva}

Este trabajo busca generar un procedimiento de producción de plantas, mediante mecanismo forzado, para producción en serie. Por lo anterior, se determinó la cantidad de semillas a procesar, el porcentaje de emergencia experimental de plantas se igualó a $100 \%$ (total deseado); la cantidad de semillas que no generó plántulas constituyó una proporción $(\%)$ del total deseado, factor de corrección que suma semillas al total deseado para determinar la cantidad total de semillas a seleccionar en terreno. Al recolectar inicialmente las semillas se seleccionó las aparentemente viables, juzgando por su integridad física, forma y calibre.

Las semillas se sometieron al tiempo de escariación con $\mathrm{HCl}(\mathrm{c})$. Según lo determinado para cada especie. Posteriormente estas se sometieron a inducción con GA3 a concentración aumentada a $800 \mathrm{ppm}$ para cubrir un mejor rango de respuesta. Luego de la escariación, en la imbibición en solución de GA3, se seleccionó las semillas que decantaron en el período máximo de 4 horas (información por experiencia); las que se plantaron en almacigueras como ya se ha descrito. Se determinó el tiempo de emergencia máxima de plántulas y la cantidad de plántulas emergidas (\%) para cada especie, como medidas de homogeneidad. Las plántulas se transplantaron a bolsa con sustrato estándar. La producción obtenida se comparó con la normal de algunas especies multiplicadas en el mismo vivero en el año anterior; naturalmente sin tratamiento pregerminativo, información entregada desde CONAF-Copiapó. El resultado se enfocó en explicar el cambio en el volumen de producción de plantas independiente del proceso natural y para efectos de evaluar si la producción podía ser sostenible y sustentable.

\section{Tratamiento estadístico}

Para cada especie se comparó el valor del tiempo de máxima emergencia de plántulas, con y sin inducción hormonal, mediante prueba t para muestras pareadas; en la cual se calcula el índice p. El indicador de ganancia en tiempo de germinación se calculó como el porcentaje representativo de la diferencia absoluta de valores con y sin GA3 en relación al valor sin aplicación de hormona. El indicador de ganancia en cantidad de plántulas se calculó como el porcentaje representativo de la diferencia absoluta de valores con y sin GA3, en relación al valor con GA3. Para todas las comparaciones, la significancia se determinó calculándose el índice $\mathrm{p}$ antes mencionado, $\mathrm{p}<0,05$ $(\alpha=5 \%)$ significó que las medidas comparadas son significativamente diferentes. 


\section{Resultados y Discusión}

\section{Germinación natural de las especies de plantas seleccionadas}

Las semillas de especies seleccionadas fueron, en general, de testa dura, compacta e impermeable; lo que permite su sobreviviencia en condiciones de aridez en Atacama. Por lo anterior, estas semillas tienden a tener largos períodos de latencia y germinación en clima árido, con poca agua e irregularmente disponible. La germinación que se realiza en el vivero se diferencia de la natural absoluta en que se mantiene la humedad en el sustrato mediante riego diario. El resto de las condiciones ambientales es la misma para la región; esta última es la condición mínima para germinar semillas sin tratamiento especial. Comparando el tiempo de germinación de las especies en condición natural (vivero) inducida (Tabla 1), se obtuvo que el tratamiento pregerminativo disminuyó significativamente el tiempo de germinación entre 44 y $65 \%$ en la temporada agosto-septiembre (primavera). Por su parte, en la inducción hecha en la temporada mayojunio (invierno), la inducción disminuyó el tiempo solo en A. capensis, $P$. chilensis y A. caven; estas tres especies muestran los más largos tiempos de germinación; en cambio, para S. molle, A. saligna y L leucocephala, la germinación natural fue estadísticamente similar a la inducida. Estas tres últimas especies son conocidas por tener buena germinación natural entre las especies locales, lo que podría confirmar que tienen mejor respuesta; representando mejor adaptación fisiológica al clima de la región de Copiapó que las tres especies anteriores.
El cambio de la respuesta invernal significa que la respuesta fisiológica vegetal cambió, disminuyendo la capacidad de respuesta germinativa de las plantas (según la especie naturalmente). Sin embargo, a pesar de la variación de respuesta germinativa, todas las especies respondieron significativamente bien a la inducción pregerminativa en primavera. El hecho general es poder obtener más rápidamente las plantas con el proceso de inducción, en promedio aritmético, comparando con la germinación en condición natural, se logró adelantar (valores \% negativos) la obtención de plantas en 52,8\% induciendo en primavera y en 23,3\% haciéndolo en invierno. La comparación entre las inducciones estacionales (primavera como referencia e invierno) muestran que la obtención de plantas en invierno fue en mayor tiempo (porcentajes positivos) que en primavera, con un promedio aritmético de $61,2 \%$.

\section{Germinación Inducida}

\section{Emergencia de plántulas en agosto-septiembre}

La temperatura promedio en la estación estuvo entre 24 y $30^{\circ} \mathrm{C}$, normal para la temporada, con precipitación histórica de $5 \mathrm{~mm}$ anuales, aun cuando no se registró precipitación efectiva en la estación ni en el plano del valle. En la respuesta de las especies tratadas (Tabla 2) con el efecto inductivo completo en primavera, el tiempo de escariación registrado fue el asociado con la mayor producción de plántulas; para cada especie se registró el tiempo de la máxima emergencia de plántulas con y sin efecto del GA3 (concentración de efecto máximo observado). Salvo

Tabla 1. Comparación de tiempos de germinación natural e inducida de especies arbóreas silvestres del Valle de Copiapó, Región de Atacama, Chile.

\begin{tabular}{|c|c|c|c|c|c|c|}
\hline \multicolumn{7}{|c|}{ Tiempo de Germinación (días) } \\
\hline \multirow{2}{*}{ Especie } & \multirow{2}{*}{$\frac{\text { Natural }}{\mathrm{NP}}$} & \multicolumn{2}{|c|}{ Inducida } & \multicolumn{3}{|c|}{ (\%) - Significancia } \\
\hline & & IP & IO & IP-NP & IO-NP & IO-IP \\
\hline S. molle & $25 \pm 5$ & $14 \pm 3,5$ & $24 \pm 5,7$ & $-44 *$ & $-4 \mathrm{~ns}$ & $71 *$ \\
\hline A. capensis & $37,8 \pm 5$ & $16 \pm 3,1$ & $18 \pm 2,3$ & $-58 *$ & $-52 *$ & $13 \mathrm{~ns}$ \\
\hline P. chilensis & $30,8 \pm 4$ & $13 \pm 3,1$ & $22 \pm 1,5$ & $-58 *$ & $-29 *$ & $69 *$ \\
\hline A. caven & $40,5 \pm 5$ & $14 \pm 2,5$ & $25 \pm 3,5$ & $-65^{*}$ & $-38 *$ & $79 *$ \\
\hline A. saligna & $22 \pm 5$ & $13 \pm 2,5$ & $25 \pm 3,5$ & $-36^{*}$ & $-14 \mathrm{~ns}$ & $79 *$ \\
\hline L. leucocephala & $15,8 \pm 3$ & $7 \pm 1,2$ & $11 \pm 2,9$ & $-58 *$ & $-30 \mathrm{~ns}$ & $57 *$ \\
\hline
\end{tabular}

S.: Schinus, A.: Acacia, P.: Prosopis, L.: Leucaena. NP: tiempo de germinación natural de primavera, IP: germinación inducida agosto-septiembre, IO: germinación inducida mayo-junio. $*=p<0,05, * *=p<0,01)$, ns $=$ no significativo. 
Tabla 2. Respuesta de especies arbóreas silvestre del Valle de Copiapó al efecto inductivo por escariación y con la hormona GA3 en la temporada agosto-septiembre.

\begin{tabular}{|c|c|c|c|c|c|c|c|}
\hline \multirow{2}{*}{ Especie } & \multirow{2}{*}{ Siembra } & \multirow{2}{*}{$\begin{array}{l}\text { Tiempo } \mathrm{HCl} \\
\text { (min) }\end{array}$} & \multirow{2}{*}{ GA3 } & \multirow{2}{*}{$\begin{array}{l}\text { Máxima emergencia plántulas } \\
\text { (días) }\end{array}$} & \multirow{2}{*}{$\begin{array}{l}\text { Emergencia plantas } \\
(\%)\end{array}$} & \multicolumn{2}{|c|}{ Ganancia (\%) } \\
\hline & & & & & & GT & GP \\
\hline \multirow{2}{*}{ S. molle } & \multirow{2}{*}{ Agosto } & \multirow{2}{*}{7} & - & $22 \pm 2^{\mathrm{A}}$ & $15 \pm 3^{\mathrm{A}}$ & \multirow{2}{*}{36,4} & \multirow{2}{*}{80,0} \\
\hline & & & + & $14 \pm 4^{\mathrm{B}}$ & $75 \pm 3^{B}$ & & \\
\hline \multirow{2}{*}{ A. capensis } & \multirow{2}{*}{ Agosto } & \multirow{2}{*}{16} & - & $23 \pm 3^{\mathrm{A}}$ & $30 \pm 5^{\mathrm{C}}$ & \multirow{2}{*}{30,4} & \multirow{2}{*}{68,4} \\
\hline & & & + & $16 \pm 3^{\mathrm{B}}$ & $95 \pm 4^{\mathrm{D}}$ & & \\
\hline \multirow{2}{*}{ P. chilensis } & \multirow{2}{*}{ Agosto } & \multirow{2}{*}{7} & - & $20 \pm 3^{\mathrm{A}}$ & $40 \pm 5^{\mathrm{C}}$ & \multirow{2}{*}{35,0} & \multirow{2}{*}{57,9} \\
\hline & & & + & $13 \pm 3^{\mathrm{B}}$ & $95 \pm 5^{\mathrm{D}}$ & & \\
\hline \multirow{2}{*}{ A. caven } & \multirow{2}{*}{ Septiembre } & \multirow{2}{*}{15} & - & - & 0 & \multirow{2}{*}{ ND } & \multirow{2}{*}{0} \\
\hline & & & + & $14 \pm 3^{\mathrm{B}}$ & $30 \pm 5^{\mathrm{C}}$ & & \\
\hline \multirow{2}{*}{ A. saligna } & \multirow{2}{*}{ Septiembre } & \multirow{2}{*}{15} & - & $24 \pm 4^{\mathrm{A}}$ & $60 \pm 4^{\mathrm{E}}$ & \multirow{2}{*}{37,0} & \multirow{2}{*}{33,3} \\
\hline & & & + & $13 \pm 3^{\mathrm{B}}$ & $80 \pm 6^{\mathrm{F}}$ & & \\
\hline \multirow{2}{*}{ L. leucocephala } & \multirow{2}{*}{ Septiembre } & \multirow{2}{*}{$X$} & - & $10 \pm 3^{\mathrm{B}}$ & $60 \pm 2^{\mathrm{E}}$ & \multirow{2}{*}{30,0} & \multirow{2}{*}{67} \\
\hline & & & + & $7 \pm 1^{\mathrm{C}}$ & $100 \pm 0^{\mathrm{D}}$ & & \\
\hline
\end{tabular}

$\mathrm{X}=$ no se aplicó el tratamiento por no ser necesario. $\mathrm{ND}=$ no determinable, el valor de referencia es cero. Uso de GA3: GA3 no aplicado y (+): GA3 aplicado. Ganancia en tiempo (GT): T = tiempo, GT $(\%)=((\mathrm{T}(-)-\mathrm{T}(+)) / \mathrm{T}(-)) 100,(-)$ : Ganancia en producción de plantas, $\mathrm{P}=$ plantas: $\mathrm{GP}(\%)=((\mathrm{P}(+)-\mathrm{P}(-)) / \mathrm{P}(-)) 100$. Para los valores en cada columna, letras A $-\mathrm{F}$ indican similaridad estadística con $*=p<0,05$.

A. caven, que se analiza a parte, con la aplicación de GA3 las restantes especies fluctuaron entre 30 (L. leucocephala) y $36,4 \%$ (S. molle) de ganancia en tiempo (días) con ganancia de producción de plántulas con valores entre 33,3 (A. saligna) y $80 \%$ (S. molle). La ganancia en tiempo entre especies no fue estadísticamente significativa ( $\mathrm{p}>>0,05)$.

Acacia caven tuvo comportamiento particular, ya que no germinó en el mayor lapso de tiempo de emergencia inducida de las otras especies de este ensayo. Sin embargo, se obtuvo que semillas de $A$. caven no inducidas germinaron posterior e irregularmente, produciendo hasta un máximo de $10 \%$ de plántulas en el tiempo estadístico de 40,5 \pm 5 días.

Considerando las especies, se calculó un índice (promedio aritmético) de 33,7\% de ganancia en tiempo; acompañando a un índice de 45,3\% de aumento en la producción de plantas por inducción, ambos con alta variabilidad, reflejando diferentes respuestas fisiológicas.

Los datos muestran que, aún con porcentaje de incremento variable; el procedimiento de inducción evaluado, siempre aumentó la tasa de producción de plantas. Este comportamiento puede proyectarse en efectos de mejoramiento de tiempo de germinación y cantidad de producción de plantas desde resultado irregular en condiciones naturales a producción masiva en menor tiempo y producción relativamente controlable.

\section{Emergencia de plántulas en mayo-junio}

La temperatura promedio en esta estación fue entre 14 y $23^{\circ} \mathrm{C}$, también normal para la temporada, no se registró precipitación efectiva en el plano del valle. En la respuesta de las especies tratadas (Tabla 3) con el efecto inductivo completo, el tiempo de escariación indicado fue el asociado con la mayor producción de plántulas (desde la Tabla 2); para cada especie se registró el tiempo de la máxima emergencia de plántulas con y sin efecto del GA3. También, salvo A. caven, la aplicación de GA3 las restantes especies mostraron ganancia en tiempo (días) de germinación entre 4,3\% no significativo (según el error estadístico de los datos) (S. molle) y $50 \%$ (L. leucocephala); con ganancia de producción de plántulas entre 50\% (S. molle) y $87,5 \%$ ( $P$. chilensis). La ganancia en tiempo entre especies no fue estadísticamente significativa. 
Tabla 3. Respuesta de especies arbórea silvestres del Valle de Copiapó al efecto inductivo por escariación y con la hormona GA3 en la temporada mayo-junio.

\begin{tabular}{|c|c|c|c|c|c|c|c|}
\hline \multirow{2}{*}{ Especie } & \multirow{2}{*}{ Siembra } & \multirow{2}{*}{$\begin{array}{l}\text { Tiempo } \mathrm{HCl} \\
\text { (min) }\end{array}$} & \multirow{2}{*}{ GA3 } & \multirow{2}{*}{$\begin{array}{l}\text { Máxima emergencia plántulas } \\
\text { (días) }\end{array}$} & \multirow{2}{*}{$\begin{array}{c}\text { Emergencia plantas } \\
(\%)\end{array}$} & \multicolumn{2}{|c|}{ Ganancia (\%) } \\
\hline & & & & & & GT & GP \\
\hline \multirow{2}{*}{ S. molle } & \multirow{2}{*}{ Junio } & \multirow{2}{*}{7} & No & $23 \pm 4^{\mathrm{A}}$ & $25 \pm 5^{\mathrm{A}}$ & \multirow{2}{*}{4,3} & \multirow{2}{*}{50,0} \\
\hline & & & $\mathrm{Si}$ & $24 \pm 6^{\mathrm{A}}$ & $50 \pm 3^{\mathrm{B}}$ & & \\
\hline \multirow{2}{*}{ A. capensis } & \multirow{2}{*}{ Mayo } & \multirow{2}{*}{16} & No & $25 \pm 2^{\mathrm{A}}$ & $10 \pm 1^{\mathrm{C}}$ & \multirow{2}{*}{28,0} & \multirow{2}{*}{66,7} \\
\hline & & & $\mathrm{Si}$ & $18 \pm 2^{\mathrm{B}}$ & $30 \pm 4^{\mathrm{A}}$ & & \\
\hline \multirow{2}{*}{ P. chilensis } & \multirow{2}{*}{ Junio } & \multirow{2}{*}{7} & No & $33 \pm 3 \mathrm{C}$ & $10 \pm 3 \mathrm{C}$ & \multirow{2}{*}{33,3} & \multirow{2}{*}{87,5} \\
\hline & & & $\mathrm{Si}$ & $22 \pm^{\mathrm{A}}$ & $80 \pm 5^{\mathrm{D}}$ & & \\
\hline \multirow{2}{*}{ A. caven } & \multirow{2}{*}{ Mayo } & \multirow{2}{*}{15} & No & - & - & \multirow{2}{*}{ ND } & \multirow{2}{*}{ ND } \\
\hline & & & $\mathrm{Si}$ & $25 \pm 4^{\mathrm{A}}$ & $20+4^{A}$ & & \\
\hline \multirow{2}{*}{ A. saligna } & \multirow{2}{*}{ Mayo } & \multirow{2}{*}{15} & No & $29 \pm 6^{\mathrm{A}}$ & $10 \pm 4^{\mathrm{C}}$ & \multirow{2}{*}{13,7} & \multirow{2}{*}{66,} \\
\hline & & & $\mathrm{Si}$ & $25 \pm 4^{\mathrm{A}}$ & $30 \pm 8^{A}$ & & \\
\hline \multirow{2}{*}{ L. leucocephala } & \multirow{2}{*}{ Mayo } & \multirow{2}{*}{$\mathrm{X}$} & No & $22 \pm 3^{A}$ & $10 \pm 4^{\mathrm{C}}$ & \multirow[t]{2}{*}{50,0} & \multirow[t]{2}{*}{66,7} \\
\hline & & & $\mathrm{Si}$ & $11 \pm 3^{\mathrm{D}}$ & $30 \pm 9^{\mathrm{A}}$ & & \\
\hline
\end{tabular}

$\mathrm{X}=$ no se aplicó el tratamiento por no ser necesario. ND = no determinable, el valor de referencia es cero. Ganancia en tiempo, $\mathrm{t}=$ tiempo: $\mathrm{GT}(\%)=((\mathrm{T} 0-\mathrm{Tt}) / \mathrm{T} 0) 100$. Ganancia en producción de plantas, $\mathrm{P}=$ plantas: $\mathrm{GP}(\%)=((\mathrm{Pt}-\mathrm{P} 0) / \mathrm{Pt}) 100$. Para los valores en cada columna, letras A - D indican similaridad estadística con * $=p<0,05$.

Tabla 4. Producción comparativa en vivero de especies arbóreas silvestres del Valle de Copiapó, antes y después del tratamiento inductivo pregerminativo, en la temporada agosto-septiembre.

\begin{tabular}{lcc}
\hline & \multicolumn{2}{c}{ Total de plantas producidas en vivero (agosto-octubre) } \\
\cline { 2 - 3 } Especie & 2011 & 2012 \\
& Sin inducción & Con inducción \\
\hline S. molle & 800 & 4.000 \\
P. chilensis & 500 & 5.000 \\
L. leucocephala & 800 & 5.000 \\
A. saligna & 500 & 2.000 \\
Total & 2.600 & 16.000 \\
Meta anual* & 10.000 & 10.000 \\
Producción según meta (\%) & $26 \%$ de la meta & $60 \%$ sobre la meta \\
Efecto sobre producción efectiva (\%) & - & 83,8 \\
\hline
\end{tabular}

* Comunicación personal CONAF.

Semillas de A. caven sin tratamiento con GA3, al igual que en la temporada anterior, no germinaron en el mayor lapso de tiempo de emergencia inducida en las otras especies. Sin embargo, también se obtuvo que las semillas mencionadas germinaron irregularmente, produciendo también hasta $10 \%$ de plantas en $40,5 \pm 5$ días.

Considerando las especies, se calculó un índice (promedio aritmético) de 53\% de ganancia de tiempo de germinación; acompañando a un índice de $50 \%$ de aumento en la producción de plantas por inducción, ambos con alta variabilidad; en esta situación, reflejando diferentes respuestas fisiológicas.

Nuevamente, y para esta estación climática, los datos muestran que, aún con porcentaje de incremento variable; en esta temporada, la inducción forzada siempre aumentó la tasa de producción de plantas; apoyando una proyección similar a la indicada para la temporada anterior, que prevé la posibilidad de 
mejorar notablemente la producción regular de plantas arbóreas nativas.

\section{Evaluación del ensayo de producción}

En la producción masiva de plantas, se comparó la producción de algunas especies tratadas antes y después del proyecto (Tabla 4), desde datos del vivero correspondiente a la temporada de producción normal (primavera-verano). Según lo anterior, la producción 2011 en el vivero; sumando montos parciales de cuatro especies en común con las del proyecto fue de 2.600 plantas con meta anual de 10.000 unidades para Copiapó (com. pers. CONAF). La producción por inducción de semillas en el vivero fue de 16.000 plantas por semana, con la misma meta anual. Desde esta comparación se obtuvo que la producción en 2011 fue $26 \%$ de la meta y la producción con el tratamiento pregerminativo inductivo fue $60 \%$ sobre la meta. El efecto del tratamiento fue un aumento de $83.8 \%$ sobre la producción efectiva de plantas del año anterior.

Sumando a lo anterior las plantas producidas en el vivero de CRIDESAT-UDA, en una semana se pudo producir 25.000 plantas, incluyendo siete especies arbóreas silvestres presentes en el Valle de Copiapó; con estos datos, la proyección de producción anual se calculó en 12.000 .000 de unidades de plantas silvestres del Valle de Copiapó, para un caso de producción continua.

Las especies escogidas habitan en el Valle de Copiapó, entre $80 \mathrm{~km}$ al sur y $150 \mathrm{~km}$ al norte; estas han disminuido su cobertura en la región, asociado al desarrollo de la población humana. En zonas áridas este tipo de especie se ha estado usando, generalmente, con propósito doméstico e industrial principalmente, como forraje, material de construcción y combustible, como ocurre en otras zonas áridas (Sepúlveda et al., 2008). Este trabajo está orientado -como ya se indicó- a hacer de la multiplicación de las especies silvestres una actividad independiente del proceso natural y, por tanto, posible de ser transformada a actividad productiva controlada en el grado necesario. Por lo anterior, el foco de este trabajo no es el tema ecológico; aunque, indudablemente la estandarización de protocolos de producción de plantas con fines no naturales, implica que se puede beneficiar la conservación natural de flora regional importante. Incluso, se puede fomentar la reforestación y forestación de áreas del territorio con especies producidas $a d$ hoc con bajo costo de obtención. Lo anterior hace que sea también importante estudiar los mecanismos biológicos para mejorar la producción regional de plantas. Por otra parte, se hace importante que la producción masiva y acelerada de plantas se incluya como un sistema de intervención equilibrante entre tasa de uso y mantención de flora silvestre.

Plantas de zonas áridas pueden presentar semillas con barreras físicas y/o fisiológicas a la germinación (Valfré-Giorello et al., 2012). Las especies estudiadas respondieron bien a la escariación e inducción hormonal, indicando la existencia de estas barreras naturales. Pero las barreras físicas son, básicamente, un mecanismo de prevención de la deshidratación natural de semillas; aunque igualmente afectan negativamente la capacidad de imbibición (Moreno et al., 2008). Por lo anterior, el ciclo biológico natural se hace más lento que la tasa de desaparición por usufructuo de poblaciones vegetales.

La comparación del tiempo de germinación por inducción completa y en estado natural, muestran que el proceso de inducción fue significativo en disminuir el tiempo de germinación. La comparación entre efectos estacionales primavera e invierno muestra que la ganancia en tiempo de germinación fue mayor en primavera. Lo anterior, apunta al estado fisiológico de las semillas influenciado por la estación; pero, finalmente, siempre implicó aumento en la eficiencia de producción de plantas según lo que se busca en este trabajo.

La ganancia de tiempo inducida en la germinación, en ambas estaciones, puede asociarse al efecto de la hormona GA3 y al clima, básicamente a la temperatura; la condición climática de primavera induce naturalmente a la producción de GA3 en las semillas. Así, en primavera, la proporción de GA3, especialmente en relación al ácido abscisico (hormona "del invierno") es mayor que en otoño (Tize y Zeiger, 2013). Siendo la escariación un factor constante; cualquiera sea la estación, la concentración artificial experimental de GA3 satura a las semillas por sobre la natural, pudiendo producir el efecto máximo de inducción hormonal de la germinación; pero, sin dejar de depender en parte de la respuesta fisiológica estacional de las semillas.

Comparando el efecto de los procesos de inducción (escariación y hormonal) en la respuesta de tiempo de máxima emergencia de plántulas entre primavera e invierno; se tiene que para S. molle, A. capensis y L. leucocephala, la 
respuesta de las semillas fue similar $(\mathrm{p}=0,6)$ y para $P$. chilensis, A. caven y A. saligna el tiempo de máxima emergencia de plántulas fue más significativo (menor) $(\mathrm{p}=0,036)$ en primavera. Por otra parte, comparando la respuesta neta de emergencia (máxima) de plantas, la producción fue significativamente mayor $(\mathrm{p}=0,03)$ desde las semillas inducidas en todas las especies, para ambas estaciones y siempre mayor en primavera que en invierno. Lo anterior indica que, al menor tiempo de germinación máxima se asocia mayor producción de plantas. Entonces, la escariación con ácido fuerte y la imbibición con GA3 fue eficiente en mejorar la tasa de producción artificial masiva de todas las especies ensayadas. Incluso, el bajo aumento de producción de plantas de Acacia caven significó en sí mismo un $100 \%$ de efecto sobre la germinación inicial cero. Este método es ya mencionado como adecuado en aplicación efectiva a flora leñosa de Argentina (Araoz y Delongo, 2006; Masini et al., 2012). Hay otros métodos como el uso de agua caliente (Doll et al., 2013); no probado en este trabajo. La escarificación puede romper la dormancia endógena (Yang et al., 2007), estimular la germinación en cantidad y velocidad (Kelly y van Staden, 1985; Pei-Sheng et al., 2008) y corrobora la observación de Travlos et al. (2007) sobre que el procedimiento puede resultar en la producción de plantas relativamente alta (sic).

La diferencia estacional en el comportamiento germinativo y de emergencia de plántulas se explicaría por la diferencia climática, teniendo la temperatura un rol importante como fue determinado para especies del bosque chaqueño, zona árida Argentina (Funes et al., 2009; Zalazar et al., 2009). Por características de Atacama y del Valle de Copiapó en particular, se puede postular que la temperatura ambiental explicaría la diferencia (disminución) de producción invernal de plantas de la región. Lo anterior hace necesario el estudio de condiciones de germinación y morfofisiología, tratamientos hormonales y nutrición, para generar tratamientos pregerminativos; especialmente en el Valle de Copiapó.

Por otra parte, la germinación también depende de características inherentes a la especie; similar a la asincronía observada en este trabajo. Cuevas y Figueroa (2007) describen germinación natural asincrónica en especies nativas amenazadas de Juan Fernández. Por otra parte, considerando la morfología en estudios pregerminativos de flora nativo del Chaco Serrano, se informa que la morfología de semillas, color del tegumento (indicador de daño por insectos), el tamaño del árbol y el peso de la semilla estaría relacionado con la capacidad germinativa (Valfré-Giorello et al., 2012). En este trabajo el efecto general observado, tanto de la escariación como de la inducción hormonal sobre las especies ensayadas, fue similar a efectos descritos en aumentar la velocidad de germinación de semillas y la tasa de germinación. Por ejemplo, Leucaena leucocephala en Atacama tuvo alta y homogénea germinación en cuatro días, similar a lo descrito por Hermosillo et al. (2008) para la misma especie (3 días) en México.

El resultado de este trabajo, aplicado a la producción masiva efectiva de especies de Atacama, en un corto período permitió superar la meta anual de CONAF con una factibilidad de alta proyección teórica anual.

Las plantas generadas quedaron depositadas en el vivero "El Pretil" de CONAF y en el vivero ubicado en CRIDESAT de la Universidad de Atacama. Estas últimas fueron ubicadas en poblaciones de la ciudad de Copiapó y entregadas a iniciativas de instituciones de la Región de Atacama, incluida la Universidad de Atacama, para usarse en campañas de arborización en Comunas de la Región (Copiapó, Chañaral y Diego de Almagro) como una forma de colaborar con forestación y reforestación. Todo el proceso sería de muy bajo costo si se masifica; obedeciendo a la idea de transmitir una metodología sencilla para que interesados de la región dispongan de una posibilidad de fuente de trabajo con especies que normalmente en condiciones naturales no germinan ni rápido ni homogéneamente ni con producción de grandes masas simultáneas.

\section{Conclusión}

La aplicación de tratamiento de escariación e inducción de germinación con GA3 aumentó la germinación y la tasa de producción de especies vegetales arbóreas silvestres de Atacama, quedando pendiente determinar el efecto del cambio térmico estacional. El comportamiento de las especies frente a la metodología de inducción, puede usarse para calcular un procedimiento con alta productividad potencial anual de plantas silvestres y nativas. Se buscan métodos de producción masiva que sean independientes de la estación climática y con producción de plantas altamente 
homogéneas, para los diversos usos posibles. Este resultado se limita al clima árido del Valle de Copiapó, para aplicarlo en otras zonas se debe determinar la respuesta de las plantas frente al tratamiento de inducción combinado naturalmente con las condiciones edafoclimáticas. El tratamiento significó ganancia en tiempo y producción de todas las especies de plantas del ensayo; comportamiento que se hace previsible para efecto de transferir la metodología a la población interesada y mejorar la producción de plantas desde natural a masiva artificial.

\section{Agradecimientos}

Este trabajo fue financiado por el Fondo de Innovación para la Competitividad, del Gobierno Regional de Atacama (2012-2013), Proyecto FICGA3 de CRIDESAT-Universidad de Atacama y con aporte de la Dirección de Investigación de la Universidad de Atacama, mediante el Proyecto DIUDA 22266 (2014). Se agradece la gestión de los colaboradores, especialmente en el apoyo de la gestión efectiva del trabajo realizado en el vivero de CONAF-Copiapó.

\section{Literatura Citada}

Alvarado, A.; Levet, O.

2014. Manual de protocolos de producción de especies utilizadas por el programa de arborización CONAF. Ed. Maval Ltda., Chile, 177 pp.

Araoz, S.D.; Del Longo, O.T.

2006. Pregerminative treatments to break the physical dormancy imposed for the endocarps in Ziziphusmistol Grisebach. Universidad Nacional de Santiago del Estero (Argentina). Quebracho, 13: 56-76.

Boeri, P.; Scelzo, L.; Ruscitti, M.; Abedini, W.; Marinucci, L. 2000. Biotécnicas aplicadas a especies forestales nativas. Investigación agraria. Sistemas y Recursos Forestales, (9) $1: 31-44$.

Camus, $\mathrm{P}$.

2004. Los bosques y la minería del Norte Chico, S. XX. Un mito en la representación del paisaje chileno. Historia, 37 (II): 289-310.

Cuevas, J.G.; Figueroa, J.A.

2007. Seed germination of species of the Juan Fernández Archipiélago under laboratory conditions. Gayana Bot., 64 (1): 60-80.

Doll, U.; Fredes, M.; Soto, C.

2013. Efecto de distintos tratamientos pregerminativos sobre la germinación de seis especies nativas de la región mediterránea de Chile. Idesia (Chile), 31 (3): 71-77.

Funes, G.; Díaz, S.; Vanier, P.

2009. La temperatura como principal determinante de la germinación en especies del Chaco seco de Argentina. Ecol. Austral, 19: 129-138.

Gutierrez, B.; Quintero, P.; Nieto, V.; Murillo, O.

2003. Enfoques cooperativos para el mejoramiento genético y la conservación de recursos forestales en Chile, Colombia y Costa Rica. Invest. Agrar. Sist. Rec. For., 12 (3): 111-122.

Hermosillo, G.; Aguirre, J.; Alonso, R.; Ortega, C.; Gómez y Magaña, R.

2008. Métodos inductivos para maximizar la germinación de semilla de germoplasma nativo en vivero para sistemas silvopastoriles en Nayarit, México. Zootecnia Trop., 26 (3): 355-358.

Kelly, K.M. y van Staden, J.

1985. Effect of acid scarification on seed coat structure, germination and seedling vigour of Aspalathus linearis. $J$. Plant Physiol., 121 (1): 37-45.
McCarty, J.P.

2001. Ecological consequences of recent climate change. Conservation Biology, 15 (2): 320-331.

Masini, A.C.A.; Rovere, A.E. y Pérez, D.R.

2012. Requerimientos pregerminativos de dos especies leñosas: Anarthrophyllum capitatum Sorarú y Anarthrophyllum elegans (Gillies ex Hook. \& Arn.) F. Philippi. Quebracho, 20 (2): 85-96.

Marinucci, L.; Ruscitti, M. y Abedini, W.

2004. Morfogénesis in vitro de leguminosas forestales nativas de la República Argentina. Rev. FCA UNCUYO, 105 (2): 27-36.

Milbau, A.; Graae, B. J.; Shevtsova, A. y Nijs, I.

2009. Effects of a warmer climate on seed germination in the subartic. Annals of Botany, 104 (2): 287-296.

Moreno, F.; Plaza, G.A. y Magnistsky, S.V.

2008. Effect of the seed coats on germination of rubber (Hevea brasiliensis Muell.) seeds. Agron. Colomb., 24 (2): 290-295.

Pei-Sheng, M.; Yu-Hong, W.; Xin-Guo, W.; Jia-Jie, L. y Ying, H. 2008. Conditions and stimulation for Germination in Glycyrrhiza uralensis Fisch. Agric Sci China, 7 (12): 1438-1444.

Phillipi, E.A.

1860. Viage* al Desierto de Atacama (*sic). Hecho de orden del gobierno de Chile en el verano 1853-54. Ed. Librería de Eduardo Anton. 270 pp.

Sepúlveda, B.; Mendieta, G.; Morgan, M. y Tume, P.

2008. Capparis scabrida (Capparaceae) (sapote) woodland characterization in northern Perú. Geographia Technica, 2: 122-131.

Tize, L.; Zeiger, E.

2010. Plant Phisiology. 5a ed, Sinauer Associates Inc., Publishers Sunderland, Massachusetts USA. 781 p.

Travlos, I.S.; Economou, G. y Karamanos, A.I.

2007. Germination and emergence of the hard seed coated Tylosema esculentum (Burch), a schreib in response to different pre-sowing seed treatments. J. Arid Environ, 68: 501-507.

Uribe, M. y Cifuentes, L.G.

2004. Aplicación de técnicas de cultivo in vitro en la propagación de Legrandia concinna. Bosque (Valdivia), 25 (1): 129-135. 
Valfré-Giorello, T.A.; Ashworth, L. y Renison, D.

2012. Patrones de germinación de semillas de Sebastiania commersoniana (Baillon) Smith \& Downs (Euphorbiaceae), árbol nativo del Chaco Serrano de interés en restauración. Ecol. Austral, 22: 92-100.

Vilela, A.E. y Ravetta, D.A.

2001. The effect of seed scarification and soil-media on germination, growth, storage, and survival of seedlings of five species of Prosopis L. (Mimosaceae). J. Arid Environ., 48 (2): 171-184.

Yang, Q-H; Ye, W-H y Yin, X-J.

2007. Dormancy and germination of Areca triandra seeds. Sci. Hort., 113 (1): 107-111.

Zalazar, M.; Funes, G. y Venier, M.P.

2009. Factores que afectan la germinación de Justicia squarrosa Griseb, forrajera nativa de la región chaqueña de la Argentina. Agriscientia, XXVI (1): 1-6. 